\title{
Dirac concentrations in Lotka-Volterra parabolic PDEs
}

\author{
Benoît Perthame* Guy Barles ${ }^{\dagger}$
}

September 19, 2018

\begin{abstract}
We consider parabolic partial differential equations of Lotka-Volterra type, with a non-local nonlinear term. This models, at the population level, the darwinian evolution of a population; the Laplace term represents mutations and the nonlinear birth/death term represents competition leading to selection.

Once rescaled with a small diffusion, we prove that the solutions converge to a moving Dirac mass. The velocity and weights cannot be obtained by a simple expression, e.g., an ordinary differential equation. We show that they are given by a constrained Hamilton-Jacobi equation. This extends several earlier results to the parabolic case and to general nonlinearities. Technical new ingredients are a $B V$ estimate in time on the non-local nonlinearity, a characterization of the concentration point (in a monomorphic situation) and, surprisingly, some counter-examples showing that jumps on the Dirac locations are indeed possible.
\end{abstract}

Key-Words: Integral parabolic equations, adaptive dynamics, asymptotic behavior, Dirac concentrations, population dynamics.

AMS Class. No: 35B25, 35K57, 49L25, 92D15

\section{Introduction}

This paper is devoted to study the asymptotic behavior of solutions to parabolic Lotka-Volterra equations. They describe the dynamics of a population density $n(t, x)$ which expands (or decays) with a growth rate $R(x, I(t))$ which changes sign. In the theory of adaptive evolution ([27, 24, 25, 26. 17, 11]), the parameter $x$ denotes a physiological "trait" and $I(t)$ an environmental unknown shared by the total population and which is used as a nutrient. The population can use the nutrient $I(t)$ differently, depending upon the trait $x$, which makes that certain fitter traits should emerge, modifying the environment and thus allowing other traits to emerge. This mechanism uses mutations in the population that we model here by a mere diffusion; but more realistic integral kernels can be handled as well, [18, 12]. This elementary modeling leads to the equations

$$
\left\{\begin{array}{l}
\frac{\partial}{\partial t} n_{\varepsilon}-\varepsilon \Delta n_{\varepsilon}=\frac{n_{\varepsilon}}{\varepsilon} R\left(x, I_{\varepsilon}(t)\right), \quad x \in \mathbb{R}^{d}, t \geq 0, \\
n_{\varepsilon}(t=0)=n_{\varepsilon}^{0} \in L^{1}\left(\mathbb{R}^{d}\right), \quad n_{\varepsilon}^{0} \geq 0,
\end{array}\right.
$$

${ }^{*}$ Université Pierre et Marie Curie-Paris 6, UMR 7598 LJLL, BC187, 4, place Jussieu, F-75252 Paris 5, and Institut Universitaire de France. Email: perthame@ann.jussieu.fr

${ }^{\dagger}$ Laboratoire de Mathématiques et Physique Théorique, CNRS UMR 6083, Fédération Denis Poisson, Université François Rabelais, Parc de Grandmont, 37200 Tours, France. Email: barles@lmpt.univ-tours.fr 


$$
I_{\varepsilon}(t)=\int_{\mathbb{R}^{d}} \psi(x) n_{\varepsilon}(t, x) d x
$$

The function $\psi$ is given and measures the 'predation' of individuals with trait $x$ on the environment. Notice that we have rescaled the problem in order to include the idea that mutations are small (or rare) thanks to the small parameter $\varepsilon>0$. Such models, together with related asymptotic, can be derived form individual based stochastic processes in the limit of large populations; we refer to [13, 14]. Also the problem without diffusion (a coupled infinite system of elementary differential equations) is interesting from the point of view of large time behavior; we expect that the dynamics concentrates on large times and several related results can be found in the literature, see [16, 28] for instance.

Our purpose is to show that, under various assumptions on the rate $R(x, I)$, the population $n_{\varepsilon}$ concentrates as a Dirac mass (or a sum of Dirac masses), a mathematical way to express that well identified species emerge from the adaptive landscape defined by the rate $R$. The interesting feature being to describe the dynamics of these Dirac masses. Here, we perform this analysis in a completely rigorous manner. But the idea to analyze adaptive dynamics in those terms, and the formalism, goes back to [18, where a well founded biological system (the chemostat) was studied. In [6], we performed a rigorous asymptotic analysis in the case of integral operators and with linear dependency on the environmental unknown $I(t)$. The case of a system, for a population with adults and juveniles, was studied in [12].

Although related, the situation of reaction-diffusion systems, as they arise in combustion, is quite different. The simple Fisher-KPP equation may serve as a model, which amounts to use $R=1-n_{\varepsilon}$ in (11), and is known to lead to the propagation of a front. This means that in the limit $\varepsilon \rightarrow 0$, the solution converges typically to either 0 (uncolonized region) or to 1 (fully colonized region), and the transition occurs on the "front", i.e., a hypersurface which dynamics can be described by the level set of a solution of a Hamilton-Jacobi Equation (see [5, 7, 8, 20, 29, 22]). Therefore, the limiting objects are, geometrically at least, very different in the cases of reaction-diffusion equations and of LotkaVolterra equations. This is the reason why in the later case a new type of equation, the constrained Hamilton-Jacobi equation, occurs to focus on the isolated points of the Dirac locations. The theory of viscosity solutions to Hamilton-Jacobi equations occurs naturally in our derivation because a phase occurs naturally; it is quite elaborate now and general introductory references are [1, 15, 3, 19, 21]. But a large part of the present paper uses other general ideas that can be read without knowledge of this notion.

The paper is organized as follows. We first state (section 21) simple and general results and in particular the convergence result to a Dirac mass for dimension one in "monomorphic situations". These results are completed by $B V$ bounds on $I_{\varepsilon}(t)$ (section 3 ) and by the asymptotic analysis through constrained Hamilton-Jacobi equations (section 4), in any dimension. Consequences are drawn in section 5, they imply the concentration of $n_{\varepsilon}$ as a sum of Dirac masses in a general multidimensional setting. Section 6 is devoted to counterexamples to the continuity of $I(t)$ (the limit of $I_{\varepsilon}$ ) and of the dirac locations, and to "smallness" conditions implying continuity. Several further results are presented afterwards: the monomorphic situation is completed in section 7 , the case of several environmental variables is treated in section 8 ,

\section{Assumptions and main results}

In this section, we give assumptions for the coefficients and data arising in (11)-(2) and state the main convergence result in a particularly simple case. More general results follow from the analysis and 
proof we perform later; these results, which are more technical, are stated along the paper.

We assume that there are two constants $\psi_{m}, \psi_{M}$ such that

$$
0<\psi_{m} \leq \psi \leq \psi_{M}<\infty, \quad \psi \in W^{2, \infty}\left(\mathbb{R}^{d}\right)
$$

The quantity $R$ is called the invasion exponent because it describes the ability of the individuals of trait $x$ to invade the population with environmental state $I(t)$. We assume that there are two constants $0<I_{m} \leq I_{M}<\infty$ such that

$$
\min _{x \in \mathbb{R}^{d}} R\left(x, I_{m}\right)=0, \quad \max _{x \in \mathbb{R}^{d}} R\left(x, I_{M}\right)=0,
$$

and there exists a constant $K>0$ such that, for any $x \in \mathbb{R}^{d}, I \in \mathbb{R}$,

$$
-K \leq \frac{\partial R}{\partial I}(x, I)<-K^{-1}<0, \quad \sup _{I_{m} / 2 \leq I \leq 2 I_{M}}\|R(\cdot, I)\|_{W^{2, \infty}\left(\mathbb{R}^{d}\right)} \leq K .
$$

We will also use the assumption

$$
n_{\varepsilon}^{0} \in L^{\infty}\left(\mathbb{R}^{d}\right), \quad \nabla n_{\varepsilon}^{0} \in L^{1}\left(\mathbb{R}^{d}\right) \quad \text { and } \quad I_{m} \leq \int_{\mathbb{R}^{d}} \psi(x) n_{\varepsilon}^{0}(x) d x \leq I_{M},
$$

and the notation

$$
\varrho_{\varepsilon}(t)=\int_{\mathbb{R}^{d}} n_{\varepsilon}(t, x) d x
$$

Notice that the assumption (3) and the bound $I_{m} \leq \int_{\mathbb{R}^{d}} \psi(x) n_{\varepsilon}(t, x) d x \leq I_{M}$ imply

$$
\frac{I_{m}}{\psi_{M}}=: \rho_{m} \leq \int_{\mathbb{R}^{d}} n_{\varepsilon}(t, x) d x \leq \rho_{M}:=\frac{I_{M}}{\psi_{m}} .
$$

One can have in mind the particular but more intuitive example

$$
\psi \equiv 1, \quad R(x, I)=b(x) Q_{1}(I)-d(x) Q_{2}(I),
$$

with $Q_{i} \in C^{1}(\mathbb{R})$ for $i=1,2$, and

$$
\begin{gathered}
Q_{1}^{\prime}(\cdot)<0, \quad Q_{2}^{\prime}(\cdot)>0, \quad Q_{i}>0 . \\
b \geq b_{m}>0, \quad d \geq d_{m}>0 \text { and } b, d \in W^{2, \infty}\left(\mathbb{R}^{d}\right) .
\end{gathered}
$$

We recall that we have the following existence result and a priori bounds (here $C$ denotes various constants which maybe different from line to line)

Theorem 2.1 With the assumptions (3)-(6) and $I_{m}-C \varepsilon^{2} \leq I_{\varepsilon}(0) \leq I_{M}+C \varepsilon^{2}$, there is a unique solution $n_{\varepsilon} \in C\left(\mathbb{R}^{+} ; L^{1}\left(\mathbb{R}^{d}\right)\right)$, to equation (1)-(2), and it satisfies,

$$
I_{m}-C \varepsilon^{2} \leq I_{\varepsilon}(t) \leq I_{M}+C \varepsilon^{2} .
$$


A proof of existence can be found, for instance, in [16] and we do not recall it. The uniform bound can also be also be found in [6, 28] (according to [16] the lower bound is unessential because they are indirect ways to guarantee non-extinction a posteriori, we keep it here for the sake of simplicity). Section 3 gives another and stronger uniform bound in time, a uniform $B V$ bound on $I_{\varepsilon}(t)$. Here, we just indicate the derivation of the upper bound in (11). We have

$$
\begin{aligned}
\frac{d}{d t} \int_{\mathbb{R}^{d}} \psi(x) n_{\varepsilon}(t, x) d x & =\varepsilon \int_{\mathbb{R}^{d}} n_{\varepsilon}(t, x) \Delta \psi d x+\frac{1}{\varepsilon} \int_{\mathbb{R}^{d}} n_{\varepsilon}(t, x) R\left(x, I_{\varepsilon}(t)\right) \\
& \leq C_{1} \varepsilon I_{\varepsilon}(t)+\frac{1}{\varepsilon} I_{\varepsilon}(t) \max _{x \in \mathbb{R}^{d}} \frac{R\left(x, I_{\varepsilon}(t)\right)}{\psi(x)}
\end{aligned}
$$

and, from the assumption (5), the right hand side becomes negative as soon as $I_{\varepsilon}(t)$ overpasses $I_{M}+\frac{C_{1} \psi_{M}}{K} \varepsilon^{2}$ and the result follows.

We can state a very simple version of our results in the simple case when dimension $d$ is equal to 1 and when, typically, the function $R(x, I)$ is monotone in $x$.

Theorem 2.2 (Dimension $d=1$ ) We assume (3)-(6), the technical condition on $n_{\varepsilon}^{0}$ in Theorem 4.1 below, and

$$
\forall I_{m}<I<I_{M} \text { there is a unique } X(I) \in \mathbb{R} \text { such that } R(X(I), I)=0 .
$$

Then, the solution $n_{\varepsilon}(t)$ to equation (1)-(2) converges in the weak sense of measures (see also Remark 3.4 below)

$$
n_{\varepsilon_{k}}(t) \underset{k \rightarrow \infty}{\longrightarrow} \varrho(t) \delta(x-\bar{x}(t))
$$

and we have

$$
\bar{x}(t)=X(I(t)), \quad R(\bar{x}(t), I(t))=0,
$$

and the pair $(\bar{x}(t), I(t))$ satisfies the constrained Hamilton-Jacobi equation given later on (see Section 4).

When $R(x, I)$ has the special form insuring uniqueness in Theorem 4.1, then the full family $n_{\varepsilon}$ converges.

Such a population is called monomorphic because a single trait is represented asymptotically. This is the general situation with a single environmental variable $I(t)$ (this is called the Competitive Exclusion Principle, [17]). To go further, several environmental variables can be introduced (see [18, 26] and our results in Section 8).

\section{$3 \quad$ BV estimates on $I_{\varepsilon}(t)$}

As a first step in our analysis of the limit $\varepsilon \rightarrow 0$ in (1), we prove strong convergence of $I_{\varepsilon}(t)$. Therefore, we complete result of Theorem 2.1 by the:

Theorem 3.1 With the assumptions (3)-(6), we have additionally to the uniform $L^{1}$ bound (11), the local uniform $B V$ and sub-Lipschitz bounds

$$
\frac{d}{d t} I_{\varepsilon}(t) \geq-\varepsilon C+e^{-K_{2} t / \varepsilon} \int \psi(x) n_{\varepsilon}^{0}(x) \frac{R\left(x, I_{\varepsilon}^{0}\right)}{\varepsilon},
$$




$$
\frac{d}{d t} \varrho_{\varepsilon}(t) \geq-C t+\int(1+\psi(x)) n_{\varepsilon}^{0}(x) \frac{R\left(x, I_{\varepsilon}^{0}\right)}{\varepsilon}
$$

where $C$ and $K_{2}$ are positive constants.

Consequently the extracted limits satisfy that $I(t)$ is nondecreasing as soon as there exist a constant $C$ independent of $\varepsilon$ such that

$$
\int \psi(x) n_{\varepsilon}^{0}(x) \frac{R\left(x, I_{\varepsilon}^{0}\right)}{\varepsilon} \geq-C e^{o(1) / \varepsilon}
$$

Then, we also have, for all $T>0$,

$$
\int_{0}^{T} \int_{\mathbb{R}^{d}} n_{\varepsilon}(t, x) R\left(x, I_{\varepsilon}(t)\right)^{2} d x d t \leq C \varepsilon(1+\varepsilon T)
$$

Remark 3.2 Our condition (14) on the initial data leaves place for a possible initial layer. In order to avoid it, one may choose well-prepared initial data that satisfy $R\left(x, I_{\varepsilon}^{0}\right)=0$ (for instance by tuning the total mass of $\left.n_{\varepsilon}^{0}\right)$. When it is not fulfilled one might observe, e.g. in numerical simulations, a fast variation of $I_{\varepsilon}(t)$ for $t \approx 0$.

Remark 3.3 From the bound (15)), we can deduce that the weak limit $n$ in $L^{\infty}\left(\mathbb{R}^{+} ; M^{1}\left(\mathbb{R}^{d}\right)\right)$ of $n_{\varepsilon_{k}}$ is supported (a.e. in $t$ ) only at points $x$ such that $R(x, I(t))$. We give a more precise result below.

Remark 3.4 Notice also that one can prove following the same lines that the family $\int_{\mathbb{R}^{d}} n_{\varepsilon}(t, x) \Psi(x) d x$ is bounded in $B V_{\text {loc }}$ for any $W^{2, \infty}$ test-function $\Psi$. The extracted limits $n_{\varepsilon_{k}}$ therefore converge a.e. in time and not merely in $w-L^{\infty}\left(\mathbb{R}^{+} ; w-M^{1}\left(\mathbb{R}^{d}\right)\right)$.

Remark 3.5 The interested reader can check that the proof below also extends previous results of [28, 16] for the continuous differential system

$$
\frac{\partial}{\partial t} n=n R(x, I(t)), \quad I(t)=\int_{\mathbb{R}} n(t, x) d x .
$$

When $R$ is monotonic in $I$ and assuming there is a unique $\bar{x}$ such that $0=R\left(\bar{x}, I_{M}\right)=\max _{x} R\left(x, I_{M}\right)$, then as $t \rightarrow \infty$, we have $n(t) \rightarrow \rho_{M} \delta(x-\bar{x})$ with $I_{M}=\rho_{M} \psi(\bar{x})$.

Proof. We begin with the proof for $I_{\varepsilon}$, then we show (15) and finally indicate the variants for the proving the result on $\varrho_{\varepsilon}$.

Concerning $I_{\varepsilon}$, we multiply the equation by $\psi$ and integrate over $\mathbb{R}^{d}$, this yields

$$
\frac{d}{d t} I_{\varepsilon}(t)=\varepsilon \int n_{\varepsilon}(t, x) \Delta \psi(x)+\mathcal{J}_{\varepsilon}(t)
$$

with $\mathcal{J}_{\varepsilon}(t)$ defined by

$$
\mathcal{J}_{\varepsilon}(t)=\int \frac{n_{\varepsilon}}{\varepsilon} \psi(x) R\left(x, I_{\varepsilon}(t)\right) .
$$

The integration by parts is justified because $\psi \in W^{2, \infty}$ and, for $\varepsilon$ fixed, both $n_{\varepsilon}$ and $\nabla n_{\varepsilon}$ belong to $L^{1}$ by easy a priori manipulations. 
The result relies on an estimate on $\mathcal{J}_{\varepsilon}(t)$. In the same way, we have

$$
\begin{aligned}
\frac{d}{d t} \mathcal{J}_{\varepsilon}(t)= & \int n_{\varepsilon} \Delta\left[\psi(x) R\left(x, I_{\varepsilon}(t)\right)\right] d x+\int \frac{n_{\varepsilon}}{\varepsilon^{2}} \psi(x) R\left(x, I_{\varepsilon}(t)\right)^{2} d x \\
& +\int \frac{n_{\varepsilon}}{\varepsilon} \psi(x) \frac{\partial}{\partial I} R\left(x, I_{\varepsilon}(t)\right) d x \frac{d}{d t} I_{\varepsilon}(t) .
\end{aligned}
$$

Now we use (16) to recover $\mathcal{J}_{\varepsilon}(t)$ from $\frac{d}{d t} I_{\varepsilon}(t)$ in the last term. And we notice the following properties : by (5) and (11), we have

$$
\int n_{\varepsilon} \Delta\left[\psi(x) R\left(x, I_{\varepsilon}(t)\right)\right]-\int n_{\varepsilon}(t, x) \Delta \psi(x) \int n_{\varepsilon}(t, x) \psi(x) \frac{\partial}{\partial I} R\left(x, I_{\varepsilon}(t)\right)=O(1) \geq-K_{1},
$$

Therefore, using again (5),

$$
\frac{d}{d t} \mathcal{J}_{\varepsilon}(t)=O(1)+\int \frac{n_{\varepsilon}}{\varepsilon^{2}} \psi(x) R\left(x, I_{\varepsilon}(t)\right)^{2} d x+\int \frac{n_{\varepsilon}}{\varepsilon} \psi(x) \frac{\partial}{\partial I} R\left(x, I_{\varepsilon}(t)\right) d x \mathcal{J}_{\varepsilon}(t) .
$$

But we have

$$
\int n_{\varepsilon}(t, x) \psi(x) \frac{\partial}{\partial I} R\left(x, I_{\varepsilon}(t)\right) \leq-K_{2}<0 .
$$

Using also that the second term in the right-hand side of the above equality is positive, we obtain

$$
\frac{d}{d t}\left(\mathcal{J}_{\varepsilon}(t)\right)_{-} \leq K_{1}-\frac{K_{2}}{\varepsilon}\left(\mathcal{J}_{\varepsilon}(t)\right)_{-}
$$

From this differential inequality we find

$$
\left(\mathcal{J}_{\varepsilon}(t)\right)_{-} \leq \varepsilon \frac{K_{1}}{K_{2}}+\left(\mathcal{J}_{\varepsilon}(0)\right)_{-} e^{-K_{2} t / \varepsilon} .
$$

The local $B V$ bound on $I_{\varepsilon}(t)=\int \psi(x) n_{\varepsilon}(t, x) d x$, as well as its monotonicity in the limit, follows by inserting this inequality in (16).

In order to prove (15), we ague as follows. We have from (16)

$$
\int_{0}^{T} \mathcal{J}_{\varepsilon}(t)=I_{\varepsilon}(T)-I_{\varepsilon}(0)+\varepsilon O(T)=C+\varepsilon C T .
$$

On the other hand, from (18), we also have, for some $K(t)>0$ bounded from above and from below away from 0

$$
\frac{d}{d t} \mathcal{J}_{\varepsilon}(t)=C+\int_{\mathbb{R}^{d}} \frac{n_{\varepsilon}}{\varepsilon^{2}} \psi(x) R\left(x, I_{\varepsilon}(t)\right)^{2} d x-\frac{K(t)}{\varepsilon} \mathcal{J}_{\varepsilon}(t)
$$

After integration, this gives

$$
\mathcal{J}_{\varepsilon}(t)=\mathcal{J}_{\varepsilon}(0) e^{-\int_{0}^{t} \frac{K}{\varepsilon}}+\int_{0}^{t} e^{-\int_{s}^{t} \frac{K}{\varepsilon}}\left(C+\int_{\mathbb{R}^{d}} \frac{n_{\varepsilon}(s, x)}{\varepsilon^{2}} \psi(x) R\left(x, I_{\varepsilon}(s)\right)^{2} d x\right) d s,
$$


and thus, using assumption (14) and with $\underline{K}=\min K(t)>0$, we arrive at

$$
\int_{0}^{T} \mathcal{J}_{\varepsilon}(t) d t \geq-C+\int_{s=0}^{T}\left(C+\int_{\mathbb{R}^{d}} \frac{n_{\varepsilon}(s, x)}{\varepsilon^{2}} \psi(x) R\left(x, I_{\varepsilon}(s)\right)^{2} d x\right) \frac{\varepsilon}{\underline{K}} d s .
$$

According to (20), we deduce that

$$
\int_{s=0}^{T} \int_{\mathbb{R}^{d}} \frac{n_{\varepsilon}(s, x)}{\varepsilon} \psi(x) R\left(x, I_{\varepsilon}(s)\right)^{2} d x d s \leq C+C \varepsilon T,
$$

and the result (15) follows.

The result on $\varrho_{\varepsilon}(t)=\int n_{\varepsilon}(t, x) d x$ follows similar lines. We write

$$
\frac{d}{d t} \varrho_{\varepsilon}(t)=\frac{1}{\varepsilon} \int n_{\varepsilon}(t, x) R\left(x, I_{\varepsilon}(t)\right) d x:=\mathcal{K}_{\varepsilon}(t),
$$

and

$$
\begin{aligned}
\frac{d}{d t} \mathcal{K}_{\varepsilon}(t) & =\int n_{\varepsilon} \Delta R\left(x, I_{\varepsilon}(t)\right)+\int \frac{n_{\varepsilon}}{\varepsilon^{2}} R\left(x, I_{\varepsilon}(t)\right)^{2}+\int \frac{n_{\varepsilon}}{\varepsilon} \frac{\partial}{\partial I} R\left(x, I_{\varepsilon}(t)\right) \frac{d}{d t} I_{\varepsilon}(t) \\
& \geq-K_{3}+\frac{K_{2}}{\varepsilon} \mathcal{J}_{\varepsilon}(t) \\
& \geq-K_{1}-K_{3}+\frac{K_{2}}{\varepsilon}\left(\mathcal{J}_{\varepsilon}(0)\right)_{-} e^{-K_{2} t / \varepsilon}
\end{aligned}
$$

(after using the inequality (19) for $\left.\left(\mathcal{J}_{\varepsilon}(t)\right)_{-}\right)$. This implies

$$
\mathcal{K}_{\varepsilon}(t) \geq \mathcal{K}_{\varepsilon}(0)-\left(K_{1}+K_{3}\right) t-\left(\mathcal{J}_{\varepsilon}(0)\right)_{-} .
$$

The result on $\frac{d}{d t} \varrho_{\varepsilon}(t)$ follows.

\section{Constrained Hamilton-Jacobi equation, uniqueness}

As already mentioned earlier, in the limit $\varepsilon \rightarrow 0$, the solution $n_{\varepsilon}$ to (1) converges weakly to a measure $n \in L^{\infty}\left(\mathbb{R}^{+} ; L^{1}\left(\mathbb{R}^{d}\right)\right.$ ) (see see Remark 3.4). In this section we give a general theory for describing properties of $n$. The statements of Theorem 2.2 follow from the present analysis (and consequences in next section.

We expect that $n_{\varepsilon}$ concentrates as Dirac masses

$$
n_{\varepsilon}(t, x) \rightarrow n(t, x)=\sum_{i} \varrho_{i}(t) \delta\left(x-x_{i}(t)\right) .
$$

This weak limit can be described more accurately through the phase function $\varphi_{\varepsilon}$ defined by

$$
n_{\varepsilon}(t, x)=e^{\varphi_{\varepsilon}(t, x) / \varepsilon}
$$

just as the Dirac mass at 0 is well approximated by the gaussian $\frac{1}{\sqrt{2 \pi \varepsilon}} e^{-|x|^{2} /(2 \varepsilon)}$. The description of $\varphi_{\varepsilon}$, and its limit, gives information on the measure $n$. 
Firstly, we obtain the following equation, equivalent to (1),

$$
\left\{\begin{array}{l}
\frac{\partial}{\partial t} \varphi_{\varepsilon}(t, x)=\left|\nabla \varphi_{\varepsilon}\right|^{2}+R\left(x, I_{\varepsilon}(t)\right)+\varepsilon \Delta \varphi_{\varepsilon}, \\
\varphi_{\varepsilon}(t=0, x)=\varphi_{\varepsilon}^{0}(x):=\varepsilon \ln n_{\varepsilon}^{0} .
\end{array}\right.
$$

Following [18, 6], in the limit, we obtain a viscosity solution to the constrained Hamilton-Jacobi equation

$$
\left\{\begin{array}{l}
\frac{\partial}{\partial t} \varphi(t, x)=|\nabla \varphi|^{2}+R(x, I(t)), \\
\max _{x \in \mathbb{R}} \varphi(t, x)=0, \quad \forall t>0, \\
\varphi(t=0, x)=\varphi^{0}(x) .
\end{array}\right.
$$

Theorem 4.1 Assume (3)-(6) and $\left(\varphi_{\varepsilon}^{0}\right)_{\varepsilon}$ is a sequence of uniformly bounded function in $W^{1, \infty}$ which converges uniformly to $\varphi^{0}$. Then, after extraction of a subsequence, $\left(\varphi_{\varepsilon}^{0}\right)_{\varepsilon}$ converges locally uniformly to a Lipschitz continuous viscosity solution $\varphi$ to 24). In particular, a.e. in $t$, $\operatorname{supp} n(t, \cdot) \subset\{\varphi(t, \cdot)=0\}$.

This solution is unique when assumptions (10) and

$$
R(x, I)=b(x)-d(x) Q(I), \quad \text { with } Q(I)>0 \text { increasing },
$$

or

$$
R(x, I)=b(x) Q(I)-d(x), \quad \text { with } Q(I)>0 \text { decreasing, }
$$

Because we expect that the set $\{\varphi(t, \cdot)=0\}$ is made of isolated points, we indeed expect that $n$ is a sum of Dirac masses. In one dimension this follows rigorously from Theorem 5.1 below but heuristically maximum points of $\varphi(t, \cdot)$ are indeed isolated in any dimension.

The result was proved in [6] in the case $R(x, I)=b(x)-d(x) Q(I)$ and for an integral operator instead of a diffusion. For completeness, we recall the proof and the main new ingredients. One of the difficulties being that coefficients, because of $I(t)$, are not discontinuous. This can be handled following well established arguments, see [2, 6].

Proof. Existence. In order to prove it, we show how to pass to the limit in (23). It is worth pointing out that we can do it in two ways, either by using the notion of viscosity solutions for equations with a $L^{1}$-dependence in time or, since $I_{\varepsilon}$ converges to an increasing function, by using the notion of viscosity solutions for equations with a discontinuous Hamiltonians. We do it in the second way which has the advantage to give a more precise result.

Since the functions $\varphi_{\varepsilon}$ are equi-bounded and equi-Lipschitz continuous in space, they are also equiHölder continuous in time (See [4] or [23] for proofs of this claim). Therefore the only difficulty is to pass to the limit (up to a subsequence) in the term $R\left(x, I_{\varepsilon}\right)$.

More precisely, in order to pass to the limit in this term, we have to compute the quantities (up to a subsequence)

$$
\limsup _{\substack{s \rightarrow t \\ \varepsilon^{\prime} \rightarrow 0}} I_{\varepsilon^{\prime}}(s) \quad \text { and } \liminf _{\substack{s \rightarrow t \\ \varepsilon^{\prime} \rightarrow 0}} I_{\varepsilon^{\prime}}(s)
$$

Indeed, the $x$-dependence in $R\left(x, I_{\varepsilon}\right)$ does not cause any problem since the functions $x \mapsto R(x, I)$ are Lipschitz continuous in $x$ and, on the other hand, the function $R(x, I)$ is decreasing in $I$ for any $x$ which allows to reduce the computation of these limsup and liminf to $I_{\varepsilon^{\prime}}$ and not $R\left(x, I_{\varepsilon^{\prime}}\right)$. 
To do so, we use Theorem 3.1] : if we set $\mu_{\varepsilon}:=\mathbb{1}_{\left[\varepsilon^{1 / 2}, T\right]}\left(\frac{d I_{\varepsilon}}{d t}+2 C \varepsilon\right) d t$, where, here and below, $\mathbb{1}_{[a, b]}$ denotes the indicator function of $[a, b]$, then the $\mu_{\varepsilon}$ 's are bounded (positive) measures on $(0, T)$ for any $T>0$, if $\varepsilon$ is small enough. Therefore we can extract a subsequence such that $\left(\mu_{\varepsilon^{\prime}}\right)_{\varepsilon^{\prime}}$ converges weakly to some measure $\mu$.

If $0<t_{0}<T$ is a point such that $\mu\left(\left\{t_{0}\right\}\right)=0$, then we can also assume that $I_{\varepsilon^{\prime}}\left(t_{0}\right)$ converges to some $\alpha \in \mathbb{R}$ and we set

$$
I(t)=\alpha+\int_{\left(t_{0}, t\right)} d \mu(s) \quad \text { if } t>t_{0} \quad \text { and } \quad I(t)=\alpha-\int_{\left(t, t_{0}\right)} d \mu(s) \quad \text { if } t \leq t_{0} .
$$

Obviously, the function $I$ is an increasing function on $(0, T)$.

We are going to show that

$$
\limsup _{\substack{s \rightarrow t \\ \varepsilon^{\prime} \rightarrow 0}} I_{\varepsilon^{\prime}}(s) \leq I(t+) \quad \text { and } \quad \liminf _{\substack{s \rightarrow t \\ \varepsilon^{\prime} \rightarrow 0}} I_{\varepsilon^{\prime}}(s) \geq I(t-)
$$

We do it for $t>t_{0}$, the other case being treated analogously.

For $s$ close to $t$, we have

$$
I_{\varepsilon^{\prime}}(s)=\alpha+\int_{\left(t_{0}, t\right)} d \mu_{\varepsilon}(\tau) .
$$

If $\zeta:[0, T] \rightarrow \mathbb{R}$ is a continuous function such that $\zeta(\tau) \geq \mathbb{1}_{\left[t_{0}, t\right]}(\tau)$ on $[0, T]$, we have

$$
I_{\varepsilon^{\prime}}(s) \leq \alpha+\int_{(0, T)} \zeta(\tau) d \mu_{\varepsilon}(\tau)
$$

and passing to the limit in this inequality, we obtain

$$
\limsup _{\substack{s \rightarrow t \\ \varepsilon^{\prime} \rightarrow 0}} I_{\varepsilon^{\prime}}(s) \leq \alpha+\int_{(0, T)} \zeta(\tau) d \mu(\tau)
$$

and we conclude by applying Lebesgue's dominated convergence Theorem to a sequence $\left(\zeta_{k}\right)_{k}$ which converges to $\mathbb{1}_{\left[t_{0}, t\right]}$ in a suitable way : namely, we choose a sequence of functions such that $0 \leq \zeta_{k} \leq 1$ on $[0, T], \zeta_{k} \equiv 1$ on $\left[t_{0}, t\right]$ for any $k$ and which converge pointwise to 0 outside the interval $\left[t_{0}, t\right]$.

The proof for the liminf is the same approximating, this time, the indicator function from below. It is worth pointing out that the property $\mu\left(\left\{t_{0}\right\}\right)=0$ avoids here (and above) discussions on the behavior of the sequence at this point. We find that this liminf is larger than $\alpha+\int_{\left(t_{0}, t\right)} \zeta(\tau) d \mu(\tau)$, which is exactly $I(t-)$.

Proof. Uniqueness. Consider for instance the second case. We consider again the function

$$
\Psi(t, x)=\varphi(t, x)-b(x) \Sigma(t), \quad \Sigma(t)=\int_{0}^{t} Q(I(s)) d s .
$$

It satisfies

$$
\frac{\partial}{\partial t} \Psi(t, x)=-d(x)+|\nabla(\Psi+b(x) \Sigma(t))|^{2}
$$


On the one hand, for two different solutions with the same initial data, we define in this way two functions $\Psi_{1}$ and $\Psi_{2}$. Using the viscosity criteria, we have, at the point $x_{0}$ where the maximum is achieved

$$
\begin{aligned}
\frac{d}{d t}\left\|\Psi_{1}-\Psi_{2}(t)\right\|_{\infty} & =\left|\nabla\left(\Psi_{1}\left(x_{0}\right)+b\left(x_{0}\right) \Sigma_{1}(t)\right)\right|^{2}-\left|\nabla\left(\Psi_{2}\left(x_{0}\right)+b\left(x_{0}\right) \Sigma_{2}(t)\right)\right|^{2} \\
& =\left(\nabla \Psi_{1}\left(x_{0}\right)+\nabla b\left(x_{0}\right) \Sigma_{1}(t)+\nabla \Psi_{2}\left(x_{0}\right)+\nabla b\left(x_{0}\right) \Sigma_{2}(t)\right) \cdot\left(\nabla b\left(x_{0}\right) \Sigma_{1}(t)-\nabla b\left(x_{0}\right) \Sigma_{2}(t)\right) \\
& \leq C\left|\nabla b\left(x_{0}\right) \Sigma_{1}(t)-\nabla b\left(x_{0}\right) \Sigma_{2}(t)\right| \\
& \leq C\|\nabla b\|_{\infty}\left|\Sigma_{1}(t)-\Sigma_{2}(t)\right|
\end{aligned}
$$

On the other hand, we also have, considering the point $x_{i}$ where $\max _{x \in \mathbb{R}^{d}} \varphi_{i}(t, x)$ is achieved

$$
\begin{aligned}
0 & =\max _{x \in \mathbb{R}^{d}} \varphi_{1}(t, x)-\max _{x \in \mathbb{R}^{d}} \varphi_{2}(t, x) \\
& \leq \varphi_{1}\left(t, x_{1}\right)-\varphi_{2}\left(t, x_{1}\right) \\
& \leq b\left(x_{1}\right)\left[\Sigma_{1}(t)-\Sigma_{2}(t)\right]+\Psi_{1}\left(t, x_{1}\right)-\Psi_{2}\left(t, x_{1}\right) \\
& \leq b\left(x_{1}\right)\left[\Sigma_{1}(t)-\Sigma_{2}(t)\right]+\max _{x \in \mathbb{R}^{d}}\left[\Psi_{1}(t, x)-\Psi_{2}(t, x)\right] .
\end{aligned}
$$

Changing the indices 1 and 2 , one of the numbers $b\left(x_{1}\right)\left[\Sigma_{1}(t)-\Sigma_{2}(t)\right]$ or $b\left(x_{2}\right)\left[\Sigma_{2}(t)-\Sigma_{1}(t)\right]$ is negative and thus we have, with the notation (10),

$$
b_{m} \mid\left(\Sigma_{1}(t)-\Sigma_{2}(t)\left|\leq \max _{x \in \mathbb{R}^{d}}\right| \Psi_{1}(t, x)-\Psi_{2}(t, x) \mid .\right.
$$

Together, the two above inequalities yield

$$
\frac{d}{d t}\left\|\left(\Psi_{1}-\Psi_{2}\right)(t)\right\|_{\infty} \leq C\left\|\left(\Psi_{1}-\Psi_{2}\right)(t)\right\|_{\infty}
$$

Uniqueness follows.

The uniqueness result can be obtained under a slightly more general assumption on $R$, namely:

for any functions $I_{1}, I_{2} \in L^{\infty}(0, T)$ and $t \in(0, T)$, the function $x \mapsto \int_{0}^{t}\left[R\left(x, I_{1}(s)\right)-R\left(x, I_{2}(s)\right)\right] d s$ has a constant sign for all $x \in \mathbb{R}^{d}$ and there exists a constant $K>0$ such that

$$
\max _{x \in \mathbb{R}^{d}}\left|\int_{0}^{t}\left[D_{x} R\left(x, I_{1}(s)\right)-D_{x} R\left(x, I_{2}(s)\right)\right] d s\right| \leq \tilde{K} \min _{x \in \mathbb{R}^{d}}\left|\int_{0}^{t}\left[R\left(x, I_{1}(s)\right)-R\left(x, I_{2}(s)\right)\right] d s\right| .
$$

The result can be stated as follows

Theorem 4.2 Assume that $\varphi^{0} \in W^{1, \infty}\left(\mathbb{R}^{d}\right)$ and that (3)-(6), (29) and the above sign condition hold. Then the problem (24) has at most one Lipschitz continuous solution.

The two conditions appearing in assumption (29) are easily checkable in the cases we emphasize in Theorem 4.1 but (a priori) they are not satisfied for general $R$ of the form $R(x, I)=b(x) Q_{1}(I)-$ $d(x) Q_{2}(I)$, even if (9), (10) hold true. Uniqueness for such general $R$ is still an open problem.

Proof. The proof follows readily the uniqueness proof of Theorem 4.1. We consider two solutions $\left(\varphi_{1}, I_{1}\right)$ and $\left(\varphi_{2}, I_{2}\right)$ and for $i=1,2$, we set

$$
\Psi_{i}(t, x)=\varphi_{i}(t, x)-\int_{0}^{t} R\left(x, I_{i}(s)\right) d s .
$$


The first part of the proof leading to (25) yields now

$$
\frac{d}{d t}\left\|\Psi_{1}-\Psi_{2}(t)\right\|_{\infty} \leq K \max _{x \in \mathbb{R}^{d}}\left|\int_{0}^{t}\left[D_{x} R\left(x, I_{1}(s)\right)-D_{x} R\left(x, I_{2}(s)\right)\right] d s\right|
$$

and the second part (cf. (26) $-(27)$ ) provides us with the inequalities

$$
\begin{aligned}
& 0 \leq \int_{0}^{t}\left[R\left(x_{1}, I_{1}(s)\right)-R\left(x_{1}, I_{2}(s)\right)\right] d s+\max _{x \in \mathbb{R}^{d}}\left[\Psi_{1}(t, x)-\Psi_{2}(t, x)\right], \\
& 0 \leq \int_{0}^{t}\left[R\left(x_{2}, I_{2}(s)\right)-R\left(x_{2}, I_{1}(s)\right)\right] d s+\max _{x \in \mathbb{R}^{d}}\left[\Psi_{2}(t, x)-\Psi_{1}(t, x)\right] .
\end{aligned}
$$

and then

$$
\min _{x \in \mathbb{R}^{d}}\left|\int_{0}^{t}\left[R\left(x, I_{1}(s)\right)-R\left(x, I_{2}(s)\right)\right] d s\right| \leq \max _{x \in \mathbb{R}^{d}}\left|\Psi_{1}(t, x)-\Psi_{2}(t, x)\right|
$$

(this is where it is important that $x \mapsto \int_{0}^{t}\left[R\left(x, I_{1}(s)\right)-R\left(x, I_{2}(s)\right)\right] d s$ does not change sign). Putting together the two inequalities for $\frac{d}{d t}\left\|\Psi_{1}-\Psi_{2}(t)\right\|_{\infty}$ and $\left\|\Psi_{1}-\Psi_{2}(t)\right\|_{\infty}$ and using assumption (29) leads to (28) and uniqueness follows.

Remark 4.3 In [6], the uniqueness proof is given in full details for merely continuous solutions. Lipschitz continuity of $\varphi$ is used here to simplify the arguments (an write them a.e.) but everything can be understood in viscosity sense.

\section{$5 \quad$ Structure properties}

We now derive consequences of the constrained Hamilton-Jacobi equation in terms the concentration points.

Theorem 5.1 Assume (5). For any $\varphi^{0} \in W^{1, \infty}\left(\mathbb{R}^{d}\right)$, the solution to 24) is semi-convex in $x$ for $t>0$, i.e. for any $t>0$, there exist a $C_{s c}(t)$ such that, for any $\xi \in \mathbb{R}^{d},|\xi|=1$, we have

$$
\frac{\partial^{2}}{\partial \xi^{2}} \varphi_{\varepsilon} \geq-C_{s c}(t), \quad i=1, \ldots, d
$$

Consequently, at a maximum point $\bar{x}(t)$ of $\varphi(t, \cdot)$ in the variable $x, \varphi(t, \cdot)$ is differentiable in $x$ (but maybe not in $t$ ) and we have

$$
\nabla \varphi(t, \bar{x}(t))=0,
$$

additionally for all Lebesgue points of $I(t)$ we have

$$
R(\bar{x}(t), I(t))=0 .
$$


The following proof does not use the $B V$ property of $I(t)$, (only the strong limit $I_{\varepsilon} \rightarrow I$ is used to pass to the limit in the Hamilton-Jacobi equation), and thus might be useful for extensions of the model where $B V$ estimates are not proved as in section 8 ,

Proof. (Proof of semi-convexity) We justify the semi-convexity directly on the diffusive equation and use the notation $\varphi_{\xi}:=\frac{\partial \varphi_{\varepsilon}}{\partial \xi}, \varphi_{\xi \xi}:=\frac{\partial^{2} \varphi_{\varepsilon}}{\partial \xi^{2}}$. We have

$$
\begin{gathered}
\frac{\partial}{\partial t} \varphi_{\xi}=2 \nabla \varphi_{\varepsilon} \cdot \nabla \varphi_{\xi}+R_{\xi}\left(x, I_{\varepsilon}(t)\right)+\varepsilon \Delta \varphi_{\xi}, \\
\frac{\partial}{\partial t} \varphi_{\xi \xi}=2 \nabla \varphi_{\varepsilon} \cdot \nabla \varphi_{\xi \xi}+2\left|\nabla \varphi_{\xi}\right|^{2}+R_{\xi \xi}\left(x, I_{\varepsilon}(t)\right)+\varepsilon \Delta \varphi_{\xi \xi} .
\end{gathered}
$$

But $\left|\nabla \varphi_{\xi}\right| \geq\left|\varphi_{\xi \xi}\right|$ because $\varphi_{\xi \xi}=\nabla \varphi_{\xi} \cdot \xi$, therefore the function $w:=\varphi_{\xi \xi}$ satisfies

$$
\frac{\partial}{\partial t} w \geq 2 \nabla \varphi_{\varepsilon} \cdot \nabla w+2 w^{2}-\bar{R}+\varepsilon \Delta w
$$

with $\bar{R}$ an upper bound on $R_{\xi \xi}\left(x, I_{\varepsilon}(t)\right)$. The semi-convexity result (in fact, the semi-convex regularizing effect) follows from suitable approximation arguments (for the initial data) and the comparison with the (absolute) subsolution given by the solution of the O.D.E. $\dot{y}=2 y^{2}-\bar{R}, y(0)=-\infty$.

(Proof of $\nabla \varphi(t, \bar{x}(t))=0$ ) We prove this equality and a related preliminary result. As we have shown above, the function $\varphi$ is semi-convex and, by classical properties of semi-convex functions, $\varphi$ is differentiable at maximum points. Therefore

$$
\nabla \varphi(t, \bar{x}(t))=0 .
$$

Moreover, it is standard that for any sequence $\left(t_{k}, x_{k}\right)$ of $x$-differentiability points of $\varphi$ which converges to $(t, \bar{x}(t))$, we also have

$$
\nabla \varphi\left(t_{k}, x_{k}\right) \rightarrow 0 \text {. }
$$

From this property, we deduce that, for $h, r>0, h, r \rightarrow 0$,

$$
\frac{1}{r h} \int_{t}^{t+h} \int_{\bar{x}(t)-r}^{\bar{x}(t)+r}|\nabla \varphi(s, y)|^{2} d s d y, \frac{1}{r h} \int_{t-h}^{t} \int_{\bar{x}(t)-r}^{\bar{x}(t)+r}|\nabla \varphi(s, y)|^{2} d s d y \rightarrow 0 .
$$

Indeed, considering the first limit for instance, a straightforward change of variable yields

$$
\int_{0}^{1} \int_{-1}^{1}|\nabla \varphi(t+h \tau, x(t)+r e)|^{2} d \tau d e,
$$

and it suffices to apply Lebesgue's dominated convergence Theorem to this integral, using the above property together with the (local) Lipschitz continuity of $\varphi$.

(Proof of $R(t, \bar{x}(t)) \leq 0$ ) We come back to the equation which holds almost everywhere, and we first integrate it on rectangles $(t, t+h) \times(\bar{x}(t)-r, \bar{x}(t)+r)$. We obtain

$$
\int_{\bar{x}(t)-r}^{\bar{x}(t)+r}\left(\varphi(t+h, y)-\varphi(t, y) d s d y \int_{t}^{t+h} \int_{\bar{x}(t)-r}^{\bar{x}(t)+r} R(y, I(s)) d s d y+\int_{t}^{t+h} \int_{\bar{x}(t)-r}^{\bar{x}(t)+r}|\nabla \varphi(s, y)|^{2} d s d y .\right.
$$


But, by the semi-convexity of $\varphi$ in $x$, we have, for $y \in(\bar{x}(t)-r, \bar{x}(t)+r)$

$$
0 \geq \varphi(t, y) \geq \varphi(t, \bar{x}(t))-\frac{C_{s c}}{2}|y-\bar{x}(t)|^{2}=O\left(r^{2}\right),
$$

while we have also $\varphi(t+h, y) \leq 0$. Using these two properties in the above equality, we deduce

$$
\frac{1}{r h} \int_{t}^{t+h} \int_{\bar{x}(t)-r}^{\bar{x}(t)+r} R(y, I(s)) d s d y+\frac{1}{r h} \int_{t}^{t+h} \int_{\bar{x}(t)-r}^{\bar{x}(t)+r}|\nabla \varphi(s, y)|^{2} d s d y \leq \frac{1}{r h} O\left(r^{2}\right) .
$$

Therefore, we obtain

$$
\frac{1}{r h} \int_{t}^{t+h} \int_{\bar{x}(t)-r}^{\bar{x}(t)+r} R(y, I(s)) d s d y \leq \frac{1}{r h} O\left(r^{2}\right)
$$

and letting $r, h$ tend to zero with $r \ll h$, we conclude that at any Lebesgue point of $I$ we have

$$
R(\bar{x}(t), I(t)) \leq 0 .
$$

(Proof of $R(t, \bar{x}(t)) \geq 0$ ) To obtain the opposite inequality, we integrate on the rectangle $(t-h, t) \times$ $(\bar{x}(t)-r, \bar{x}(t)+r)$. The left-hand side is now

$$
\int_{\bar{x}(t)-r}^{\bar{x}(t)+r}\left(\varphi(t, y)-\varphi(t-h, y) d s d y \geq \int_{\bar{x}(t)-r}^{\bar{x}(t)+r} \varphi(t, y) d s d y\right.
$$

and, by the afore mentioned argument, it is larger than $O\left(r^{2}\right)$ and we are lead to

$$
\frac{1}{r h} \int_{t-h}^{t} \int_{\bar{x}(t)-r}^{\bar{x}(t)+r} R(y, I(s)) d s d y+\frac{1}{r h} \int_{t-h}^{t} \int_{\bar{x}(t)-r}^{\bar{x}(t)+r}|\nabla \varphi(s, y)|^{2} d s d y \geq \frac{O(r)}{h} .
$$

Again we let $r, h$ tend to zero with $r \ll h$ and using (32), we conclude that, at any Lebesgue point of $I$, we have

$$
Q(t, \bar{x}(t)) \geq 0
$$

Let us recall that because $I(t)$ is $B V$, it is continuous away from a countable set of discontinuity points. Hence, we can give another proof of the relation (30). Let $t_{0}$ a continuity point of $I(t)$ and let $\bar{x}\left(t_{0}\right)$ be a maximum point of $\varphi\left(t_{0}\right)$.

First, we use the viscosity subsolution criteria at such a point $\left(t_{0}, \bar{x}\left(t_{0}\right)\right)$, testing against the test function 0 . We find

$$
0 \leq R\left(\bar{x}\left(t_{0}\right), I\left(t_{0}\right)\right)+0 .
$$

To get the other inequality, we integrate in time the equation (24) on $\left(t_{0}, t_{0}+h\right)(h>0)$ at the point $x=\bar{x}\left(t_{0}\right)$ and find

$$
0 \geq \frac{1}{h} \varphi\left(t_{0}+h, \bar{x}\left(t_{0}\right)\right) \geq \frac{1}{h} \int_{s=0}^{h} R\left(\bar{x}\left(t_{0}\right), I\left(t_{0}+s\right)\right) d s .
$$

Because $t_{0}$ is a continuity point of $I\left(t_{0}\right)$, we find

$$
0 \geq \liminf _{h \rightarrow 0^{+}} \frac{\varphi\left(t_{0}+h, \bar{x}\left(t_{0}\right)\right)-\varphi\left(t_{0}, \bar{x}\left(t_{0}\right)\right)}{h} \geq R\left(\bar{x}\left(t_{0}\right), I\left(t_{0}\right)\right) .
$$

Clearly, (33) and (34) give the relation (30). 


\section{Continuity and discontinuity of $I(t)$ and $\bar{x}(t)$}

So far our results have shown that the Lagrange multiplier $I(t)$ is $B V$ and that the concentration point $\bar{x}(t)$ is unique in one dimension. It is natural to ask whether these quantities are continuous. This question is particularly relevant because it is possible to derive formally a differential equation on $\bar{x}(t)$, see [18, 6]

$$
\frac{d}{d t} \bar{x}(t)=\left(-D^{2} \varphi(t, x)\right)^{-1} \nabla_{x} R(\bar{x}(t), I(t)) .
$$

In this section, we answer negatively to this question (in general) based on analytical and numerical examples. We also give a smallness assumption implying continuity.

\subsection{An analytical counter-example}

Our analytical counterexample follows earlier ideas developed for combustion models, see [22, 29] for instance.

We choose in this section $\psi \equiv 1$, in other words $I(t)=\varrho(t)$. We first consider the simple equation

$$
\varphi_{t}=x-\varrho(t)+\left|\varphi_{x}\right|^{2} \quad \text { in }(0,+\infty) \times \mathbb{R},
$$

together with the initial data

$$
\varphi(x, 0)=\varphi_{0}(x) \text { in } \mathbb{R}
$$

and the constraint

$$
\max _{x \in \mathbb{R}} \varphi(t, x)=0 \quad \text { for any } t .
$$

In a first step, we start by solving the initial value problem for $\Psi=\varphi-\int_{0}^{t} \varrho(s) d s$ with the initial data $\varphi_{0}(x)=-x^{2}$. We look for a solution of the form

$$
a(t)(x-b(t))^{2}+c(t) .
$$

Easy computations yield

$$
\begin{cases}a^{\prime}(t)=4 a^{2}(t), & a(0)=-1, \\ -2 a(t) b^{\prime}(t)=1, & b(0)=0, \\ c^{\prime}(t)=b(t), & c(0)=0 .\end{cases}
$$

Therefore, we have the explicit coefficients and solution

$$
\begin{gathered}
a(t)=-\frac{1}{1+4 t}, \quad b(t)=\frac{t}{2}+t^{2}, \quad c(t)=\frac{t^{2}}{4}+\frac{t^{3}}{3}, \\
\Psi(t, x)=-\frac{1}{1+4 t}\left(x-\frac{t}{2}-t^{2}\right)^{2}+\frac{t^{2}}{4}+\frac{t^{3}}{3} .
\end{gathered}
$$

From this, we deduce that the solution to (35)-(37) is

$$
\varphi(t, x)=-\frac{1}{1+4 t}\left(x-\frac{t}{2}-t^{2}\right)^{2}, \quad \varrho(t)=\frac{t}{2}+t^{2} .
$$

This is consistent with the property $\varrho(t)=\bar{x}(t)=b(t)$ of Theorem 2.2 . 
In a second step, we remark that, in order to solve the problem on $\Psi=\varphi-\int_{0}^{t} \varrho(s) d s$ with initial data $-(x-\alpha)^{2}-\delta(\alpha>0)$, we have just (i) to translate the solution obtained with $\alpha=0$, (ii) to subtract $\delta-\alpha$ to take into account the new right-hand side of the equation and the initial data. Therefore this solution is

$$
-\frac{1}{1+4 t}\left(x-\alpha-\frac{t}{2}-t^{2}\right)^{2}-\delta+\alpha t+\frac{t^{2}}{4}+\frac{t^{3}}{3}
$$

Finally, we solve (35)-(37) with

$$
\varphi_{0}(x)=\max \left(-x^{2},-(x-\alpha)^{2}-\delta\right) \quad \text { in } \mathbb{R} .
$$

Since the Hamiltonian of (35) is concave, the maximum of two solutions is a solution : this is a consequence of the Barron-Jensen approach ([9, 10, see also [2]). Thus the solution of this problem is

$$
\max \left(-\frac{1}{1+4 t}\left(x-\frac{t}{2}-t^{2}\right)^{2},-\frac{1}{1+4 t}\left(x-\alpha-\frac{t}{2}-t^{2}\right)^{2}-\delta+\alpha t\right) \quad \text { for } t \leq \bar{t}:=\frac{\delta}{\alpha}
$$

with $\varrho(t)=\frac{t}{2}+t^{2}$, while

$$
\max \left(-\frac{1}{1+4 t}\left(x-\frac{t}{2}-t^{2}\right)^{2}+\delta-\alpha t,-\frac{1}{1+4 t}\left(x-\alpha-\frac{t}{2}-t^{2}\right)^{2}\right) \quad \text { for } t>\bar{t},
$$

with $\varrho(t)=\alpha+\frac{t}{2}+t^{2}$.

This shows the phenomena of discontinuity of $\varrho(t)$ and of the Dirac concentration point $\bar{x}(t)$.
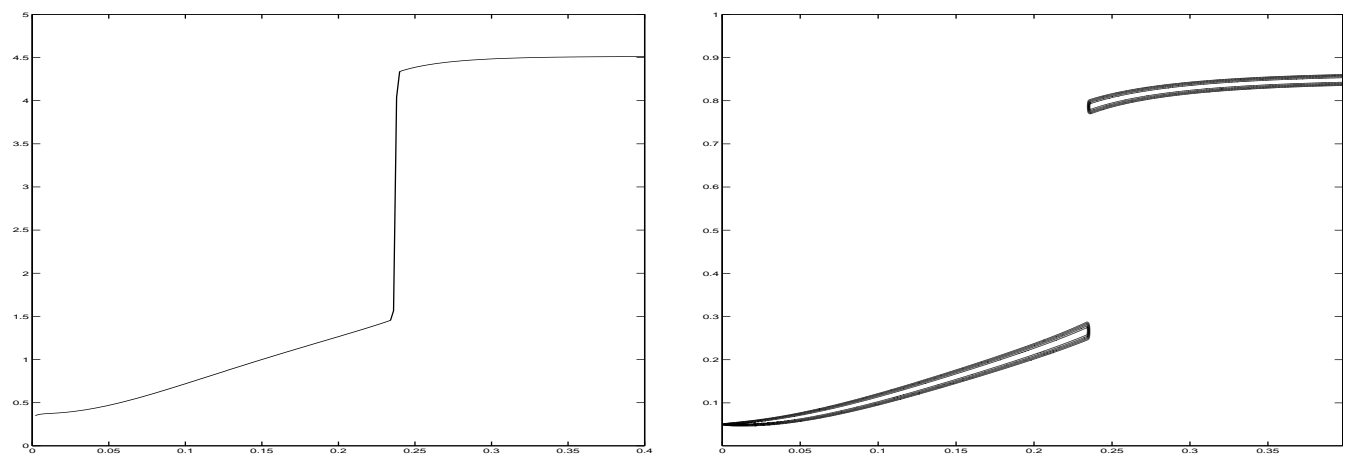

Figure 1: A numerical example of discontinuous solution in example (38). The abscissae is time. (Left) The Lagrange multiplier $\varrho(t)$. (Right) The Concentration point $\bar{x}(t)$ (in fact isovalues of the Density $\left.n_{\varepsilon}(t, x)\right)$.

\subsection{A numerical counter-example}

We give a numerical simulation of a similar phenomena which however does not use a discontinuity of the gradient to generate the discontinuities of the Lagrange multiplier and the concentration point. We use again $\psi \equiv 1(I=\varrho)$, and

$$
R(x, \varrho)=\left(x-x^{2}+3 x^{4}\right)\left(9-(1+x)^{3}\right)-\varrho .
$$



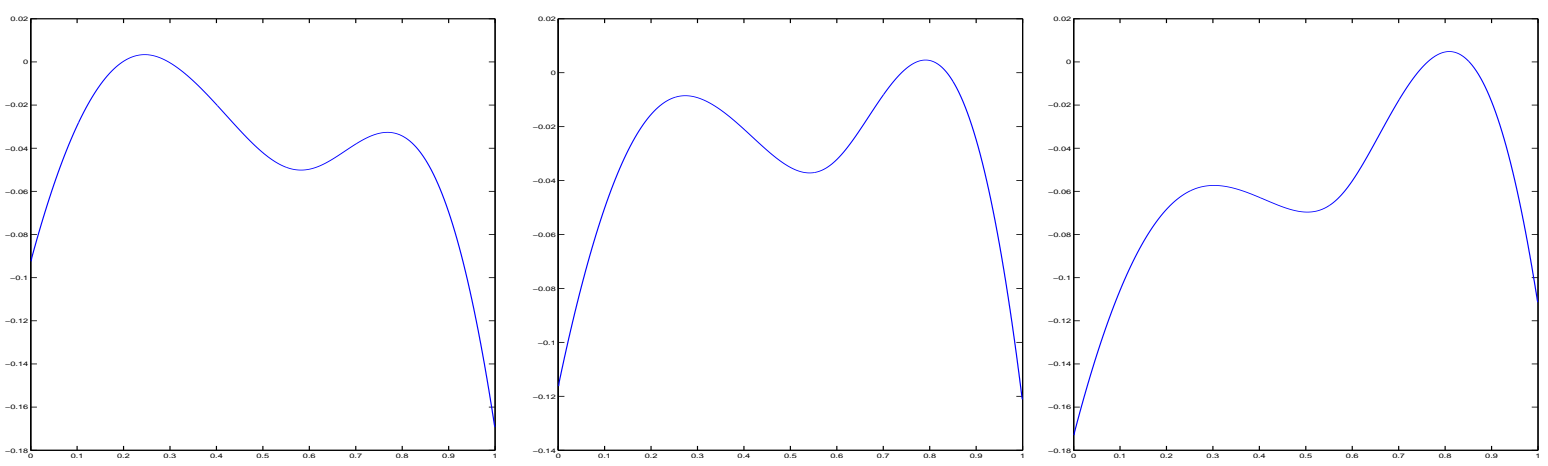

Figure 2: Discontinuity in example (38) as in Figure 1, The phase $\varphi(t, x)$ as a function of $x$ at different

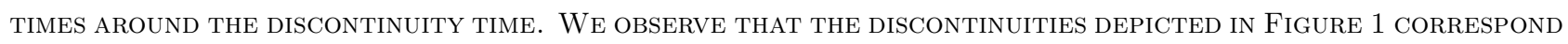
TO A SMOOTH TRANSITION ON $\varphi$.

The computations depicted in Figures 1 and 2 are performed with $0 \leq x \leq 1$ and $\varphi^{0}(x)=-|x-0.05|$. They show how a smooth change on the phase funtion $\varphi(t, x)$ along time, can produce a discontinuity of the Lagrange multiplier $I(t)$ and the concentration point $\bar{x}(t)$. A local maximum of $\varphi(t, x)$ in $x$ becomes larger as time goes and becomes the global maximum at the discontinuity time. This follows the scenario in the analytical counter-example.

\subsection{Concavity conditions for continuity}

In this section, we are interested in conditions that imply the continuity of the $I(t)$ and $\bar{x}(t)$. Because a discontinuity corresponds to a double maximum of $\varphi(t, x)$, it is natural to look for conditions implying the concavity of the solution $\varphi$. This is the purpose of the next

Theorem 6.1 Assume (5) and that $R(\cdot, I)$ is strictly concave, uniformly for bounded $I$. Then, for any $\varphi^{0} \in W^{1, \infty}\left(\mathbb{R}^{d}\right), \varphi^{0}$ uniformly concave, any solution to 24) is strictly concave and thus $\bar{x}(t)$ is continuous. Therefore, in Theorem 5.1, the equalities hold everywhere and $I(t)$ is also continuous.

Proof. Let $\varphi$ a solution of (24) and $0<\alpha<1$. We consider the function

$$
\chi(x, y, t):=\alpha \varphi(t, x)+(1-\alpha) \varphi(t, y)-\varphi(t, \alpha x+(1-\alpha) y) .
$$

The aim is to prove that $\chi(x, y, t)<0$ for any $x, y \in \mathbb{R}^{d}$ such that $x \neq y$ and for any $t$. To do so, we first show that $\chi$ is a viscosity subsolution of

$$
\chi_{t} \leq 2 K\left|\chi_{x}+\chi_{y}\right|+\frac{\chi_{x}^{2}}{\alpha}+\frac{\chi_{y}^{2}}{1-\alpha}+\tilde{R}_{\alpha}(x, y, t) \quad \text { in } \mathbb{R}^{d} \times \mathbb{R}^{d} \times(0,+\infty),
$$

where $K:=\|D \varphi\|_{\infty}$ and

$$
\tilde{R}_{\alpha}(x, y, t)=\max _{|J| \leq|| I \|_{\infty}}\{\alpha R(x, J)+(1-\alpha) R(y, J)-R(\alpha x+(1-\alpha) y, J)\}
$$

We are going to argue below as if the function $t \mapsto R(x, I(t))$ were continuous : a rigourous proof would consists in approximating $I$ by continuous functions, in proving that the corresponding $\chi$ 's satisfy the above inequality (this is also why we put a max in the definition of $\tilde{R}_{\alpha}$ : to point out the 
uniformity in $I$ ) and finally to pass to the limit. We drop these details here for the sake of simplicity in the presentation.

Consider a test function $\phi(x, y, t)$ in the viscosity sense and $(\bar{x}, \bar{y}, \bar{t})$ is a strict maximum point of $\chi(x, y, t)-\phi(x, y, t)$, we look for local maximum points of

$$
\alpha \varphi(t, x)+(1-\alpha) \varphi(t, y)-\varphi(t, z)-\frac{|\alpha x+(1-\alpha) y-z|^{2}}{\varepsilon}-\phi(x, y, t)
$$

near $(\bar{x}, \bar{y}, \bar{t})$. By classical arguments, since $(\bar{x}, \bar{y}, \bar{t})$ is a strict maximum point of $\chi(x, y, t)-\phi(x, y, t)$, there exists a sequence $\left(x_{\varepsilon}, y_{\varepsilon}, z_{\varepsilon}, t_{\varepsilon}\right)$ of maximum points of this new function which converge to $(\bar{x}, \bar{y}, \alpha \bar{x}+(1-\alpha) \bar{y}, \bar{t})$ as $\varepsilon$ tends to 0 .

If $p:=\frac{2(\alpha x+(1-\alpha) y-z)}{\varepsilon}$, standard uniqueness arguments (cf. [15]) provide the existence of $a, b, c$ (playing the roles of the $t$-derivatives of $\varphi$ at respectively $\left.\left(x_{\varepsilon}, t_{\varepsilon}\right),\left(y_{\varepsilon}, t_{\varepsilon}\right),\left(z_{\varepsilon}, t_{\varepsilon}\right)\right)$ such that

$$
\begin{gathered}
a \leq\left|p+\frac{D_{x} \phi\left(x_{\varepsilon}, y_{\varepsilon}, t_{\varepsilon}\right)}{\alpha}\right|^{2}+R\left(x_{\varepsilon}, I\left(t_{\varepsilon}\right)\right), \\
b \leq\left|p+\frac{D_{y} \phi\left(x_{\varepsilon}, y_{\varepsilon}, t_{\varepsilon}\right)}{1-\alpha}\right|^{2}+R\left(y_{\varepsilon}, I\left(t_{\varepsilon}\right)\right), \\
c \geq|p|^{2}+R\left(z_{\varepsilon}, I\left(t_{\varepsilon}\right)\right),
\end{gathered}
$$

and with $\alpha a+(1-\alpha) b-c \geq \phi_{t}\left(x_{\varepsilon}, y_{\varepsilon}, t_{\varepsilon}\right)$.

Combining these inequalities, we obtain, after straightforward computations

$$
\begin{gathered}
\phi_{t}\left(x_{\varepsilon}, y_{\varepsilon}, t_{\varepsilon}\right) \leq 2 p \cdot\left(D_{x} \phi\left(x_{\varepsilon}, y_{\varepsilon}, t_{\varepsilon}\right)+D_{y} \phi\left(x_{\varepsilon}, y_{\varepsilon}, t_{\varepsilon}\right)\right)+ \\
\left|\frac{D_{x} \phi\left(x_{\varepsilon}, y_{\varepsilon}, t_{\varepsilon}\right)}{\alpha}\right|^{2}+\left|\frac{D_{y} \phi\left(x_{\varepsilon}, y_{\varepsilon}, t_{\varepsilon}\right)}{1-\alpha}\right|^{2}+\alpha R\left(x_{\varepsilon}, I\left(t_{\varepsilon}\right)\right)+(1-\alpha) R\left(y_{\varepsilon}, I\left(t_{\varepsilon}\right)\right)-R\left(z_{\varepsilon}, I\left(t_{\varepsilon}\right)\right) .
\end{gathered}
$$

To conclude, we first remark that $|p| \leq\|D \varphi\|_{\infty}$ since $p$ is in the $x$-subdifferential of $\varphi$ at $x$, and then let $\varepsilon$ tends to 0 . Since $z_{\varepsilon} \rightarrow \alpha \bar{x}+(1-\alpha) \bar{y}$, we have

$\alpha R\left(x_{\varepsilon}, I\left(t_{\varepsilon}\right)\right)+(1-\alpha) R\left(y_{\varepsilon}, I\left(t_{\varepsilon}\right)\right)-R\left(z_{\varepsilon}, I\left(t_{\varepsilon}\right)\right) \rightarrow \alpha R(\bar{x}, I(\bar{t}))+(1-\alpha) R(\bar{y}, I(\bar{t}))-R(\alpha \bar{x}+(1-\alpha) \bar{y}, I(\bar{t}))$

and we are done.

In order to prove that $\chi(x, y, t)<0$ for any $x, y \in \mathbb{R}^{d}$ such that $x \neq y$ and $t>0$, we first remark that $\chi(x, y, t) \leq 0$ for any $x, y \in \mathbb{R}^{d}$ as a consequence of a standard comparison result : indeed since $\tilde{R}_{\alpha}(x, y, t) \leq 0$ (because $R(\cdot, I)$ is concave for any $\left.I\right), \chi$ is a subsolution of the P. D. E.

$$
w_{t}=2 K\left|w_{x}+w_{y}\right|+\frac{w_{x}^{2}}{\alpha}+\frac{w_{y}^{2}}{1-\alpha} \text { in } \mathbb{R}^{d} \times \mathbb{R}^{d} \times(0,+\infty),
$$

while 0 is a solution, and $\chi(x, y, 0) \leq 0$ in $\mathbb{R}^{d} \times \mathbb{R}^{d}$ because $\varphi^{0}$ is strictly concave; therefore $\chi(x, y, t) \leq 0$ in $\mathbb{R}^{d} \times \mathbb{R}^{d} \times(0,+\infty)$.

On the other hand, if $\chi(x, y, t)=0$ at some point $(x, y, t)$, then $(x, y, t)$ is a maximum point of $\chi-0$ and the viscosity subsolution inequality reads

$$
0 \leq \tilde{R}_{\alpha}(x, y, t),
$$

a contradiction with the strict concavity of $R(\cdot, I)$, uniformly in $I$ for $I$ bounded, which implies $\tilde{R}_{\alpha}(x, y, t)<0$. 


\section{The monomorphic case: more precise statements}

We consider in this section the special case when $d=1$ and we assume a monomorphic situation according to (12) which leads us to introduce the following assumption

There exists a function $\tilde{R}(x, I)$ such that $x \mapsto \tilde{R}(x, I)$ is strictly monotone for any $I, I \mapsto \tilde{R}(x, I)$ is strictly decreasing for any $x$ and

$$
\tilde{R}(x, I)=0(\text { resp. }>0,<0) \text { if and only if } R(x, I)=0(\text { resp. }>0,<0) .
$$

A typical example of such situation was given in the introduction, see (8) with assumptions (9) $-(10)$. When $R$ is of the form $R(x, I)=b(x) Q_{1}(I)-d(x) Q_{2}(I)$. Then (39) reduces to

$$
x \mapsto \frac{b(x)}{d(x)} \text { is strictly monotone. }
$$

Indeed, one can choose $\tilde{R}(x, I)=\frac{R(x, I)}{d(x) Q_{1}(I)}=\frac{b(x)}{d(x)}-\frac{Q_{2}(I)}{Q_{1}(I)}$. This is weaker than assuming $b^{\prime}>0$ and $d^{\prime}<0$ for which $R$ satifies (39).

Theorem 7.1 Assume (10), (39) and that $\varphi^{0} \in W^{1, \infty}\left(\mathbb{R}^{d}\right)$ satisfies

$$
\max _{\mathbb{R}} \varphi^{0}(x)=0 \quad \text { and } \quad \limsup _{|x| \rightarrow+\infty} \varphi^{0}(x)<0 .
$$

For all $t>0$ except perhaps for a countable number, there exists a unique $x(t)$ such that

$$
\varphi(t, \bar{x}(t))=\max _{\mathbb{R}} \varphi(t, x)=0 .
$$

Moreover $t \mapsto x(t)$ is monotone and, with the notations $x\left(t^{+}\right)=\lim _{s \downarrow t} x(s), x\left(t^{-}\right)=\lim _{s \uparrow t} x(s)$, then

1. If $\tilde{R}$ is increasing in $x$ then $x\left(t^{+}\right)$the largest maximum point of $\varphi(t, \cdot)$ and $x\left(t^{-}\right)$is the smallest one.

2. If $\tilde{R}$ is decreasing in $x$ then $x\left(t^{+}\right)$the smallest maximum point of $\varphi(t, \cdot)$ and $x\left(t^{-}\right)$is the largest one.

Proof. Using assumption (41) and cone of dependence type properties (recall that $\varphi$ is Lipschitz continuous), we see that, for any $T>0$ and $t \in(0, T)$, the maximum of the function $x \mapsto \varphi(t, x)$ is achieved in a fixed compact subset of $\mathbb{R}$. And, by (30), if $t$ is a Lebesgue's point of $I$ (meaning here a continuity point of the increasing function $I$ ) and $\bar{x}$ is maximum of $\varphi(\cdot, t)$, we have

$$
R(\bar{x}, I(t))=0 .
$$

Therefore, by (39),

$$
\tilde{R}(\bar{x}, I(t))=0 ;
$$

we deduce that $\bar{x}$ is unique for such $t$, we denote it by $x(t)$ and, from (9) and (40), we see that $t \mapsto x(t)$ has the same monotonicity as $\tilde{R}$ since $I(t)$ is increasing.

If $t_{k} \downarrow t$, passing to the limit in $\varphi\left(t_{k}, x\left(t_{k}\right)\right)=0$, we see that $\varphi\left(t, x\left(t^{+}\right)\right)=0$. Choosing a sequence $\left(t_{k}\right)_{k}$ of continuity points of $x(\cdot)$ or equivalently of $I(\cdot)$, we have also

$$
\tilde{R}\left(x\left(t^{+}\right), I\left(t^{+}\right)\right)=0 .
$$


Now assume that $\tilde{R}$ is strictly increasing in $x$ and that there exists a maximum point $\bar{x}>x\left(t^{+}\right)$of $\varphi(t, \cdot)$. Then for $y$ close enough to $\bar{x}$ and $s>t$, using that $I\left(t^{+}\right)=\lim _{s \downarrow t} I(s)$, we have

$$
\tilde{R}(y, I(s)) \geq \tilde{R}\left(x\left(t^{+}\right), I(s)\right)+\eta,
$$

for some $\eta>0$, and therefore, using again (39), for $s$ close enough to $t$

$$
R(y, I(s)) \geq \tilde{\eta}
$$

for some $\tilde{\eta}$, which would imply that $\varphi(s, \bar{x})>0$, a contradiction. Therefore such $\bar{x}$ does not exist and actually, $x\left(t^{+}\right)$is the largest maximum point of $\varphi(t, \cdot)$.

In the same way, $x\left(t^{-}\right)$is the smallest maximum point of $\varphi(t, \cdot)$ and the case when $\tilde{R}$ is strictly decreasing follows along the same lines.

\section{Several environmental unknowns $I_{\varepsilon}^{(k)}(t)$}

Several possible extensions are possible which are more realistic and give more interesting structures. In particular the selection rate $R$ may depend on several integrals and competition may be modeled by convolution terms $([23,16,26])$. Here we study the former extension when the model contains several environmental unknowns. This means that we consider the equation

$$
\left\{\begin{array}{l}
\frac{\partial}{\partial t} n_{\varepsilon}-\varepsilon \Delta n_{\varepsilon}=\frac{n_{\varepsilon}}{\varepsilon} R\left(x, I_{\varepsilon}(t)\right), \quad x \in \mathbb{R}^{d}, t \geq 0, \\
n_{\varepsilon}(t=0)=n_{\varepsilon}^{0} \in L^{1}\left(\mathbb{R}^{d}\right), \quad n_{\varepsilon}^{0} \geq 0
\end{array}\right.
$$

and now the environment is described by a vector valued unknown $I_{\varepsilon}=\left(I_{\varepsilon}^{(1)}, \ldots, I_{\varepsilon}^{(I)}\right)$, with

$$
I_{\varepsilon}^{(i)}(t)=\int \psi^{(i)}(x) n_{\varepsilon}(t, x) d x, \quad i=1, \ldots, N .
$$

The $\psi^{(i)}(x)$ are still given positive functions (see the precise assumptions below).

We may have in mind the example when mortality and birth terms depend differently on the total population, i.e., $N=2$ and

$$
R(x, I)=b(x) Q_{1}\left(I^{(1)}(t)\right)-d(x) Q_{2}\left(I^{(2)}(t)\right) .
$$

We may also have in mind the example of the chemostat with several nutrients

$$
R(x, I)=\sum_{i=1}^{I} b_{i}(x) Q_{i}\left(I^{(i)}(t)\right)-d(x) .
$$

Our first goal is to derive the $L^{\infty}$ estimates. This uses the following assumptions; there are constants $\psi_{m}<\psi_{M}, 0<I_{m} \leq I_{M}<\infty$ such that (vector inequalities should be understood componentwise)

$$
\begin{gathered}
0<\psi_{m} \leq \psi \leq \psi_{M}<\infty, \quad \psi^{(i)} \in W^{2, \infty}\left(\mathbb{R}^{d}\right) . \\
\min _{x \in \mathbb{R}^{d}} R(x, I) \leq 0, \text { when } \min _{1 \leq i \leq N} I^{(i)} \geq I_{M},
\end{gathered}
$$




$$
\max _{x \in \mathbb{R}^{d}} R(x, I) \geq 0, \text { when } \max _{1 \leq i \leq N} I^{(i)} \leq I_{m},
$$

and there exists a constant $K>0$ such that, for any $x \in \mathbb{R}^{d}, I \in \mathbb{R}^{N}$

$$
-K \leq \frac{\partial}{\partial I^{(i)}} R(x, I)<-K^{-1}<0, \quad \sup _{I_{m} / 2 \leq I \leq 2 I_{M}}\|R(\cdot, I)\|_{W^{2, \infty}\left(\mathbb{R}^{d}\right)} \leq K .
$$

Then, an easy adaptation of the proof of Theorem 2.1 yields

Theorem 8.1 With the assumptions (44)-(47) and $I_{m}-C \varepsilon^{2} \leq I_{\varepsilon}(0) \leq I_{M}+C \varepsilon^{2}$ (componentwise), we have the uniform bound

$$
I_{m}-C \varepsilon^{2} \leq I^{(i)}(t) \leq I_{M}+C \varepsilon^{2}, \quad \forall t \geq 0, \quad \forall i=1, \ldots, N
$$

with $C$ a positive constant.

It is unclear if one can expect $B V$ estimates (and monotonicity results in the limit) on the quantities

$I_{\varepsilon}^{(i)}$. Assuming such a bound, one might follow the arguments of Section 4 and derive again the constrained H.-J. equation (24) where now $I(t)$ is a $N$ dimensional Lagrange multiplier associated with the (single) constraint $\max _{x \in \mathbb{R}^{d}} \varphi(t, x)=0$. Of course this rises several fundamental questions concerning uniqueness of the solution to this constrained H.-J. equation. Some information is clearly lost because the quantities $I^{(i)}$ are related together in that case when $n_{\varepsilon}$ concentrates on less than $N$ Dirac masses (for instance in the monomorphic case). Therefore we face here a fundamental nonuniqueness situation.

\section{References}

[1] Bardi, M., Capuzzo Dolcetta, I. Optimal control and viscosity solutions of Hamilton-JacobiBellman equations. Birkhauser, Boston, 1997.

[2] Barles, G. Discontinuous viscosity solutions of first-order Hamilton-Jacobi equations: a guided visit. Nonlinear Anal. 20 (1993), no. 9, 1123-1134.

[3] Barles, G. Solutions de viscosité et équations de Hamilton-Jacobi. Collec. SMAI, Springer-Verlag, Paris 2002.

[4] Barles, G., Biton, S., Ley, O. A geometrical approach to the study of unbounded solutions of quasilinear equations in $R^{N}$. Arch. Rational Mech. Anal. 162 (2002) 287-325.

[5] Barles, G., Evans, L. C., Souganidis, P. E. Wavefront propagation for reaction diffusion systems of PDE, Duke Math. J. 61 (1990) 835-858.

[6] Barles, G., Perthame, B. Concentrations and constrained Hamilton-Jacobi equations arising in adaptive dynamics. In Recent Developments in Nonlinear Partial Differential Equations, D. Danielli editor. To appear in Contemp. Math. (2007).

[7] Barles, G., Souganidis, P. E. A remark on the asymptotic behavior of the solution of the KPP equation. C. R. Acad. Sci. Paris Sér. I Math. 319 (1994), no. 7, 679-684. 
[8] Barles, G., Souganidis, P. E. Front propagation for reaction-diffusion equations arising in combustion theory. Asymptotic Analysis 14 (1997), 277-292.

[9] Barron, E. N., Jensen, R. Semicontinuous viscosity solutions for Hamilton-Jacobi equations with convex Hamiltonians. Comm. Partial Differential Equations 15 (1990), no. 12, 1713-1742.

[10] Barron, E. N., Jensen, R. Optimal control and semicontinuous viscosity solutions. Proc. Amer. Math. Soc. 113 (1991), no. 2, 397-402.

[11] Calcina, À, Cuadrado, S. Small mutation rate and evolutionarily stable strategies in infinite dimensional adaptive dynamics. J. Math. Biol. 48 (2004) 135-159.

[12] Carrillo, J. A., Cuadrado, S., Perthame, B. Adaptive dynamics via Hamilton-Jacobi approach and entropy methods for a juvenile-adult model. Mathematical Biosciences, vol. 205(1) (2007), $137-161$.

[13] Champagnat, N. A microscopic interpretation for adaptive dynamics trait substitution sequence models. Stoch. Processes and their Appl. vol. 116(8) (2006), 1127-1160.

[14] Champagnat, N., Ferrière, R., Méleard, S. Unifying evolutionary dynamics: from individual stochastic processes to macroscopic models. Theoretical Population Biology, 69 (2006), No. 3, $297-321$.

[15] Crandall, M. G., Ishii, H., Lions, P.-L. User's guide to viscosity solutions of second order partial differential equations, Bull. Amer. Math. Soc. 27 (1992), 1-67.

[16] Desvillettes, L., Jabin, P.-E., Mischler, S., Raoul, G. On mutation selection dynamics. Preprint (2007).

[17] Diekmann O. Beginner's guide to adaptive dynamics. Banach Center Publications 63 (2004) 47-86.

[18] Diekmann, O., Jabin, P.-E., Mischler, S., Perthame, B. The dynamics of adaptation : an illuminating example and a Hamilton-Jacobi approach, Th. Pop. Biol., 67(4) (2005) 257-271.

[19] Evans, L. C. Partial Differential Equations, Graduate Studies in Mathematics, Vol. 19, American Mathematical Society (1998).

[20] Evans L.C., Souganidis P.E. A PDE approach to geometric optics for certain reaction-diffusion equations. Indiana Univ. Math J. 38 (1989), 141-172.

[21] Fleming W. H., Soner H. M. Controlled Markov processes and viscosity solutions. Applications of Mathematics 25, Springer (1993).

[22] Freidlin, M. Functional integration and partial differential equations. Princeton Univ. Press, Princeton (1985).

[23] Génieys, S., Perthame, B. Dynamics of Nonlocal Fisher concentration points: a nonlinear analysis of Turing patterns. Preprint 2007. http://hal.archives-ouvertes.fr/hal-00128250/fr/

[24] Geritz S. A. H., Metz J. A. J., Kisdi E., Meszena G. Dynamics of adaptation and evolutionary branching. Physical Review Letters 78 (1997) 2024-2027. 
[25] Geritz, S.A.H., Kisdi, E., Meszena, G., Metz, J.A.J. Evolutionary singular strategies and the adaptive growth and branching of the evolutionary tree, Evolutionary Ecology 12 (1998) 35-57.

[26] Meszéna, G., Gyllenberg, M. Link between population dynamics and dynamics of darwinian evolution. Phys. Rev. Letters 95, 078105 (4 pages), (2005).

[27] Metz J. A. J., Geritz S. A. H., Meszna G., Jacobs F. J. A., van Heerwaarden, J. S. Adaptive dynamics, a geometrical study of the consequences of nearly faithful reproduction. Stochastic and spatial structures of dynamical systems (Amsterdam, 1995), 183-231, Konink. Nederl. Akad. Wetensch. Verh. Afd. Natuurk. Eerste Reeks, 45, North-Holland, Amsterdam, 1996.

[28] Perthame, B. Transport equations in biology. Series 'Frontiers in Mathematics', Birkhauser (2007).

[29] Souganidis, P. E. Front propagation: theory and applications, CIME course on 'Viscosity solutions', Lecture Notes in Math., Springer-Verlag, Berlin (1998). 